\title{
Iterative Receiver for Hybrid Asymmetrically Clipped Optical OFDM
}

\author{
Qi Wang, Zhaocheng Wang, Senior Member, IEEE, and Linglong Dai, Member, IEEE
}

\begin{abstract}
This paper proposes an iterative receiver to enhance the performance of hybrid asymmetrically clipped optical orthogonal frequency division multiplexing (HACO-OFDM) in optical wireless communication systems. In HACO-OFDM scheme, asymmetrically clipped optical OFDM (ACO-OFDM) and pulseamplitude-modulated discrete multitone (PAM-DMT) signals are transmitted simultaneously, which is more spectrally efficient compared with ACO-OFDM and PAM-DMT. However, the existing HACO-OFDM receiver directly recovers the signals in the frequency domain, which could not eliminate the interference thoroughly between ACO-OFDM and PAM-DMT signals and limits its performance. In our proposed receiver, the ACO-OFDM and PAM-DMT signals are detected in the frequency domain and regenerated in the time domain. After that, they are subtracted from the received signals iteratively. Thus, ACO-OFDM and PAM-DMT signals can be distinguished. By taking advantage of the signal symmetry properties of ACO-OFDM and PAM-DMT in the time domain, pairwise clipping is utilized to further reduce the effect of noise and estimation error, resulting in improved performance. In addition, unequal power allocation is proposed to guarantee that ACO-OFDM and PAM-DMT signals have similar performance in HACO-OFDM systems. Simulation results show that the proposed method provides significant signal-to-noise ratio gain over the conventional receiver for both equal and unequal power allocations at the cost of slightly increased complexity.
\end{abstract}

Index Terms-Asymmetrically clipped optical orthogonal frequency division multiplexing (ACO-OFDM), hybrid asymmetrically clipped optical orthogonal frequency division multiplexing (HACO-OFDM), iterative receiver, optical wireless communications, pulse-amplitude-modulated discrete multitone (PAM-DMT).

\section{INTRODUCTION}

$\mathbf{O}$ PTICAL wireless communications (OWC) have been emerging as a promising complementary technique to radio frequency counterpart due to their distinct advantages such as unregulated bandwidth, low power assumption, and reliable security [1]. In order to achieve high-rate transmission, orthogonal frequency-division multiplexing (OFDM) is commonly used in OWC, whereby gigabit transmission rates have been reported [2]-[5].

Manuscript received July 15, 2014; revised September 10, 2014; accepted September 13, 2014. Date of publication September 16, 2014; date of current version October 15, 2014. This work was supported by the National Key Basic Research Program of China under Grant 2013CB329203, the National Nature Science Foundation of China under Grant 61271266, the National High Technology Research and Development Program of China under Grant 2014AA01A704, and the Beijing Natural Science Foundation under Grant 4142027.

The authors are with the Tsinghua National Laboratory for Information Science and Technology, Department of Electronic Engineering, Tsinghua University, Beijing 100084, China (e-mail: qiwang11@mails.tsinghua.edu.cn; zcwang@tsinghua.edu.cn; daill@tsinghua.edu.cn).

Color versions of one or more of the figures in this paper are available online at http://ieeexplore.ieee.org.

Digital Object Identifier 10.1109/JLT.2014.2358611
In OWC systems, intensity modulation with direct detection (IM/DD) is commonly used where the transmitted electrical signal is modulated onto the instantaneous power of the optical emitter [6]. Therefore, the transmitted signal has to be realvalued and non-negative. To guarantee that the time-domain signals are real-valued, Hermitian symmetry can be imposed on the OFDM subcarriers. By adding a DC bias, the signal is forced to be non-negative and this scheme is called DC-biased optical OFDM (DCO-OFDM) [7]. DCO-OFDM is inefficient in terms of optical power since the DC bias does not carry information. Therefore, two schemes have been proposed to improve the power efficiency, namely, asymmetrically clipped optical OFDM (ACO-OFDM) and pulse-amplitude-modulated discrete multitone (PAM-DMT). In ACO-OFDM where only the odd subcarriers are used for modulation, the time domain signals are antisymmetric and can be directly clipped at zero [8]. PAM-DMT only modulates the imaginary part of each subcarrier and also allows asymmetric clipping at zero similar to that in ACO-OFDM [9]. However, ACO-OFDM and PAM-DMT only utilize half of the subcarriers or signal dimensions, resulting in an obvious spectral efficiency loss. A hybrid ACO-OFDM (HACO-OFDM) scheme is recently proposed in [10], where ACO-OFDM signals occupying odd subcarriers and PAM-DMT signals using even subcarriers are combined in the time domain for simultaneous transmission. The HACO-OFDM retains the advantage of both ACO-OFDM and PAM-DMT, that is, high power efficiency since no DC bias is needed. Besides, it improves the spectral efficiency compared with ACO-OFDM and PAM-DMT.

The clipping noise of ACO-OFDM only appears on the even subcarriers, which is orthogonal to the desired signal, while the clipping noise of PAM-DMT only falls on the real part of each even subcarrier. Therefore, the ACO-OFDM symbols on the odd subcarriers are not distorted by clipping noise and can be directly recovered by simple fast Fourier transform (FFT) at the receiver [10]. After that, the clipping noise of ACO-OFDM on the even subcarriers can be regenerated and subtracted, so that PAM-DMT signals could be recovered using the imaginary part of the even subcarriers. While the receiver proposed in [10] is simple and straightforward, it does not fully utilize the property of HACO-OFDM to eliminate the interference between ACOOFDM and PAM-DMT signals, which limits its performance.

In this paper, an iterative receiver is proposed to enhance the performance of HACO-OFDM in IM/DD based optical wireless systems, where the ACO-OFDM and PAM-DMT signals are recovered from the received signal iteratively. More specifically, the ACO-OFDM and PAM-DMT signals are detected in the frequency domain and then regenerated in the time domain. After 
that, the regenerated signals are subtracted from the received signals so that ACO-OFDM and PAM-DMT signals could be distinguished in the time domain. Based on the observation that half of the transmitted ACO-OFDM and PAM-DMT signals are zero in the time domain, pairwise clipping is utilized and half of the signals are set to zero to further reduce the effect of noise and estimation error, thus improving the system performance. In addition, unequal power allocation is proposed to guarantee that ACO-OFDM and PAM-DMT signals have similar performance in HACO-OFDM systems. Our simulations confirm that the proposed receiver could significantly improve the performance of HACO-OFDM systems with both equal and unequal power allocations.

The remainder of this paper is organized as follows. In Section II, the transceiver of HACO-OFDM is presented, while in Section III, our proposed iterative receiver is described. In Section IV, the performance of the proposed iterative receiver is compared to the conventional receiver, while the conclusions are drawn in Section V.

\section{HACO-OFDM}

In this section, we will give a brief review of ACO-OFDM and PAM-DMT and then address the basic principle of HACOOFDM.

\section{A. ACO-OFDM and PAM-DMT}

In OFDM-based OWC systems with $N$ subcarriers, the transmitted bit stream is mapped onto the complex-valued symbols, $X_{k}, k=1,2, \ldots, N-1$, according to the chosen modulation scheme, such as PAM or quadrature amplitude modulation (QAM). Hermitian symmetry is imposed on the OFDM subcarriers to guarantee that the time-domain OFDM signals are realvalued, where we have $X_{k}=X_{N-k}^{*}, k=1,2, \ldots, N / 2-1$, and the 0 -th as well as the $N / 2$-th subcarriers are set to zero. In addition, several schemes have been proposed to guarantee that the transmitted signals are non-negative such as DCO-OFDM, ACO-ODFM, PAM-DMT and HACO-OFDM.

For ACO-OFDM, only odd subcarriers are modulated. The frequency domain ACO-OFDM signals are as follows,

$$
\mathbf{X}=\left[0, X_{1}, 0, X_{3}, \ldots, X_{N / 2-1}, 0, X_{N / 2-1}^{*}, \ldots, X_{1}^{*}\right] .
$$

The time-domain OFDM signal is obtained by the inverse fast Fourier transform (IFFT) as [11]

$$
x_{\mathrm{ACO}, n}=\frac{1}{\sqrt{N}} \sum_{k=0}^{N-1} X_{k} \exp \left(j \frac{2 \pi}{N} n k\right), n=0,1, \ldots, N-1
$$

which follows a half-wave symmetry as

$$
x_{\mathrm{ACO}, n}=-x_{\mathrm{ACO}, n+N / 2}, n=0,1, \ldots, N / 2-1 .
$$

Therefore, the negative part can be clipped without any loss of information:

$$
\begin{aligned}
\left\lfloor x_{\mathrm{ACO}, n}\right\rfloor_{c} & =x_{\mathrm{ACO}, n}+i_{\mathrm{ACO}, n} \\
& = \begin{cases}x_{\mathrm{ACO}, n}, & x_{\mathrm{ACO}, n} \geq 0 ; \\
0, & x_{\mathrm{ACO}, n}<0\end{cases}
\end{aligned}
$$

for $n=0,1, \ldots, N-1$, where $i_{\mathrm{ACO}, n}$ denotes the negative clipping noise of ACO-OFDM. It has been proved in [8] that the negative clipping noise only falls on the even subcarriers, which is orthogonal to the transmitted data on the odd subcarriers. Thus, the transmitted signal on the odd subcarriers could be recovered using simple FFT operation at the receiver.

For PAM-DMT, all excluding the 0 -th and $N / 2$-th subcarriers are modulated with PAM signals. The frequency-domain PAMDMT signals are as follows,

$$
\mathbf{Y}=j\left[0, Y_{1}, Y_{2}, \ldots, Y_{N / 2-1}, 0, Y_{N / 2-1}^{*}, \ldots, Y_{1}^{*}\right]
$$

where $Y_{k}$ for $k=0,1, \ldots, N / 2-1$ denotes the real-valued PAM signal and $j=\sqrt{-1}$. After IFFT, the time-domain PAMDMT signal $y_{\mathrm{PAM}, n}$ follows a different half-wave symmetry as

$$
y_{\mathrm{PAM}, n}=-y_{\mathrm{PAM}, N-n}, n=1, \ldots, N / 2-1
$$

and we have $y_{\mathrm{PAM}, 0}=y_{\mathrm{PAM}, N / 2}=0$. Similar to ACO-OFDM, the negative part of PAM-DMT signal can also be directly clipped to $\left\lfloor y_{\mathrm{PAM}, n}\right\rfloor_{c}$ as (4) without any information loss and the negative clipping noise only falls on the real part of each subcarrier [9].

\section{B. HACO-OFDM}

Both ACO-OFDM and PAM-DMT only utilize half of the subcarriers or dimensions, resulting in a spectral efficiency loss by half. In order to improve the spectral efficiency, HACOOFDM is recently proposed in [10], whereby ACO-OFDM and PAM-DMT signals are combined to transmit simultaneously. In PAM-DMT, only the imaginary part of even subcarriers is modulated by PAM symbols to make sure that the ACO-OFDM symbols are not interfered. The clipping noise of PAM-DMT with only even subcarriers modulated is only on the real part of the even subcarriers according to [10]. The combined transmitted signal of HACO-OFDM in the time domain is given by

$$
z_{n}=\left\lfloor x_{\mathrm{ACO}, n}\right\rfloor_{c}+\left\lfloor y_{\mathrm{PAM}, n}\right\rfloor_{c}
$$

for $n=0,1, \ldots, N-1$ and DC bias is not required as in ACOOFDM and PAM-DMT.

At the receiver, the shot noise and thermal noise is usually modeled as additive white Gaussian noise (AWGN) and the received signal is given by $r_{n}=z_{n}+w_{n}, n=0,1, \ldots, N-$ 1 , where $w_{n}$ denotes the sample of AWGN [12]. The received signal is fed to the FFT block and the frequency-domain symbols are generated as $R_{k}=Z_{k}+W_{k}$ for $k=0,1, \ldots, N-1$.

The corresponding HACO-OFDM receiver architecture was proposed in [10]. The negative clipping noise of both ACOOFDM and PAM-DMT only falls on the even subcarriers. Therefore, the ACO-OFDM symbols on the odd subcarriers could be firstly detected as [10]

$$
\hat{X}_{\mathrm{ACO}, k}=\arg \min _{X \in \mathcal{S}_{\mathrm{ACO}}}\left|X-2 R_{k}\right|, k=1,3, \ldots, N / 2-1
$$

where $\mathcal{S}_{\mathrm{ACO}}$ denotes the constellation set of ACO-OFDM. The factor of 2 in (8) is due to the fact that the clipping operation 


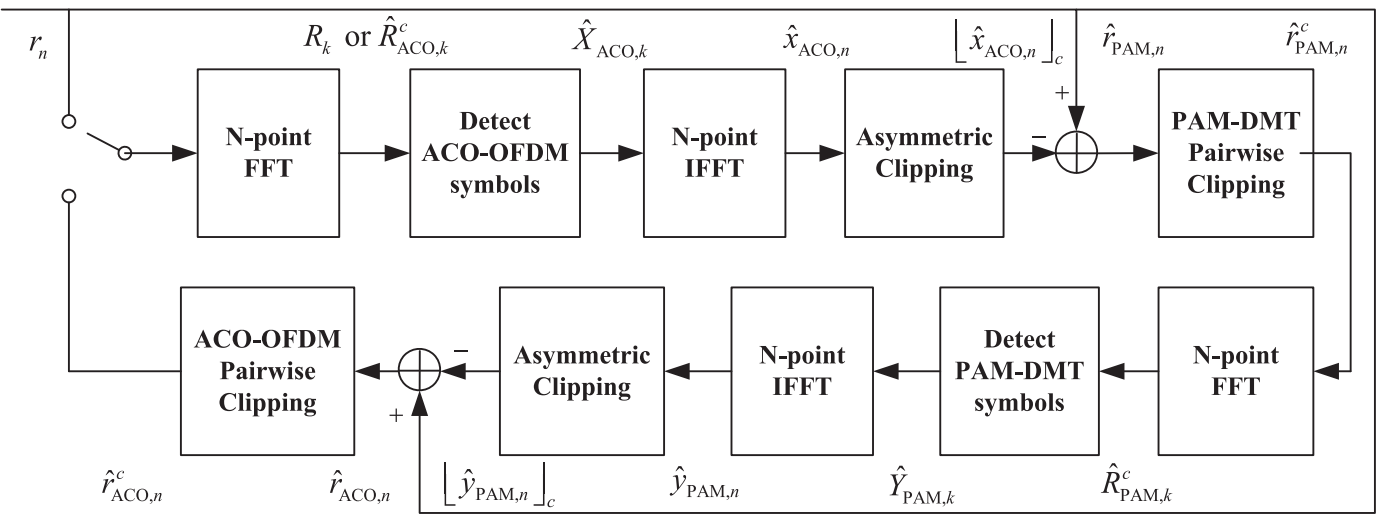

Fig. 1. Iterative receiver for HACO-OFDM.

reduces the power of ACO-OFDM symbols in the odd subcarriers by half.

The estimated transmitted time-domain ACO-OFDM signal $\left\lfloor\hat{x}_{\mathrm{ACO}, n}\right\rfloor_{c}$ could be regenerated by $\hat{X}_{\mathrm{ACO}, k}$, and the negative clipping noise of ACO-OFDM $\hat{I}_{\mathrm{ACO}, k}$ in the frequency domain could also be estimated by FFT of $\left\lfloor\hat{x}_{\mathrm{ACO}, n}\right\rfloor_{c}$. After subtracting $\hat{I}_{\mathrm{ACO}, k}$ from the received frequency-domain symbols on the even subcarriers, the PAM-DMT symbols could be readily estimated as

$$
\begin{array}{r}
\hat{Y}_{\mathrm{PAM}, k}=\arg \min _{Y \in \mathcal{S}_{\mathrm{PAM}}}\left|Y-2 \cdot \operatorname{imag}\left(R_{k}-\hat{I}_{\mathrm{ACO}, k}\right)\right|, \\
k=2,4, \ldots, N / 2-2
\end{array}
$$

where $\mathcal{S}_{\text {PAM }}$ denotes the constellation set of PAM-DMT.

\section{ITERATIVE RECEIVER FOR HACO-OFDM}

\section{A. Proposed Iterative Receiver}

While the conventional receiver proposed in [10] is simple and straightforward, it does not eliminate the interference thoroughly between ACO-OFDM and PAM-DMT signals, which limits its performance. To further improve the performance of HACO-OFDM, we propose a novel iterative receiver which reduces the interference between ACO-OFDM and PAM-DMT signals in the time domain, where pairwise clipping is inserted.

The proposed iterative receiver for HACO-OFDM is illustrated in Fig. 1, where the uppercase letters denote frequencydomain symbols and lowercase letters represent time-domain signals. In each iteration, we firstly detect ACO-OFDM symbol on the odd subcarriers by using FFT. After that, ACOOFDM time-domain signal is regenerated and subtracted from the received signal. The remaining signal is considered as the interfered PAM-DMT signal and pairwise clipping is applied to reduce the effect of noise and estimation error. The pairwise clipped PAM-DMT signal is fed to FFT module to detect PAM-DMT symbol, and we can also estimate the corresponding time-domain PAM-DMT signal. The estimated time-domain PAM-DMT signal is then subtracted from the received signal and pairwise clipping is employed to acquire more accurate ACO-OFDM signal. The receiver directly employs the received signal to detect ACO-OFDM symbol in the first iteration and the pairwise clipped ACO-OFDM signal is used in the subsequent iterations. More specifically, the design of the iterative receiver is detailed as follows.

In the first iteration, FFT is performed on the received signals and the detection of ACO-OFDM symbols is the same as (8), where $\hat{X}_{\mathrm{ACO}, k}$ for $k=1,3, \ldots, N / 2-1$ are obtained by maximum likelihood estimation. After that, the transmitted timedomain ACO-OFDM signals $\left\lfloor\hat{x}_{\mathrm{ACO}, n}\right\rfloor_{c}, n=0,1, \ldots, N-1$ could be regenerated by (2) and (4).

Unlike the conventional receiver where the ACO-OFDM symbol is subtracted in the frequency domain, the estimated time-domain ACO-OFDM signal is subtracted from the received time-domain signal $r_{n}$, resulting in the interfered time-domain PAM-DMT signal as

$$
\hat{r}_{\mathrm{PAM}, n}=r_{n}-\left\lfloor\hat{x}_{\mathrm{ACO}, n}\right\rfloor_{c}, n=0,1, \ldots, N-1 .
$$

If the receiver noise and the estimation error are considered, we can rewrite (10) as

$\hat{r}_{\mathrm{PAM}, n}=\left\lfloor y_{\mathrm{PAM}, n}\right\rfloor_{c}+w_{n}+e_{\mathrm{ACO}, n}, n=0,1, \ldots, N-1$

where $e_{\mathrm{ACO}, n}=\left\lfloor x_{\mathrm{ACO}, n}\right\rfloor_{c}-\left\lfloor\hat{x}_{\mathrm{ACO}, n}\right\rfloor_{c}$ denotes the estimation error induced by the ACO-OFDM estimation, which follows a Gaussian distribution according to the central limit theorem.

For PAM-DMT, it is notable that half of the time-domain signals are set to zero due to (6), and the remaining signals are non-negative. Therefore, for the pair of transmitted signals $\left\lfloor y_{\mathrm{PAM}, n}\right\rfloor_{c}$ and $\left\lfloor y_{\mathrm{PAM}}, N-n\right\rfloor_{c}, n=0,1, \ldots, N / 2-1$, one of them has to be zero. Similar to the algorithm proposed for ACOOFDM in [13], pairwise maximum likelihood detector is used for PAM-DMT to estimate the signals with value of zero. For $n=1, \ldots, N / 2-1$, we use the following pairwise clipping:

$$
\begin{aligned}
\hat{r}_{\mathrm{PAM}, n}^{c} & =\hat{r}_{\mathrm{PAM}, n} I_{\left\{\hat{r}_{\mathrm{PAM}, N-n} \leq \hat{r}_{\mathrm{PAM}, n}\right\}} \\
\hat{r}_{\mathrm{PAM}, N-n}^{c} & =\hat{r}_{\mathrm{PAM}, N-n} I_{\left\{\hat{r}_{\mathrm{PAM}, N-n}>\hat{r}_{\mathrm{PAM}, n}\right\}}
\end{aligned}
$$

where $I_{\{A\}}$ is an indicator function with $I_{\{A\}}=1$ if the event $A$ is true and $I_{\{A\}}=0$ otherwise. If the estimation of zero-valued signals is accurate enough, which holds under high signal-tonoise (SNR) scenarios, half of the noise and estimation error of 
ACO-OFDM could be eliminated, thus achieving a considerable performance gain. Besides, it is easy to find that $y_{\mathrm{PAM}, 0}=$ $y_{\mathrm{PAM}, N / 2}=0$ by (2) and (5), thus $\hat{r}_{\mathrm{PAM}, 0}^{c}$ and $\hat{r}_{\mathrm{PAM}, N / 2}^{c}$ are also set to zero to further reduce the noise and estimation error.

The pairwise clipped PAM-DMT signal $\hat{r}_{\mathrm{PAM}, n}^{c}$ is directly fed to the FFT block, where we have the frequency-domain symbol $\hat{R}_{\mathrm{PAM}, k}^{c}$, and the PAM-DMT symbol on the even subcarriers is estimated as

$$
\begin{aligned}
\hat{Y}_{\mathrm{PAM}, k} & =\arg \min _{Y \in \mathcal{S}_{\mathrm{PAM}}}\left|Y-2 \cdot \operatorname{imag}\left(\hat{R}_{\mathrm{PAM}, k}^{c}\right)\right|, \\
k & =2,4, \ldots, N / 2-2
\end{aligned}
$$

instead of (9).

The estimation in (14) is more accurate compared with (9) in the conventional receiver since part of the noise and estimation error are eliminated. It is used to further improve the accuracy of the estimation of the ACO-OFDM symbols in an iterative way, where the time-domain PAM-DMT signals are also regenerated and subtracted from the received signal. Then we have

$$
\hat{r}_{\mathrm{ACO}, n}=r_{n}-\left\lfloor\hat{y}_{\mathrm{PAM}, n}\right\rfloor_{c}, n=0,1, \ldots, N-1,
$$

where $\hat{r}_{\mathrm{ACO}, n}$ contains both receiver noise and the estimation error of PAM-DMT and we can rewrite (15) as

$\hat{r}_{\mathrm{ACO}, n}=\left\lfloor y_{\mathrm{ACO}, n}\right\rfloor_{c}+w_{n}+e_{\mathrm{PAM}, n}, n=0,1, \ldots, N-1$,

where $e_{\mathrm{PAM}, n}=\left\lfloor y_{\mathrm{PAM}, n}\right\rfloor_{c}-\left\lfloor\hat{y}_{\mathrm{PAM}, n}\right\rfloor_{c}$ denotes the estimation error induced by the PAM-DMT estimation. As with the ACO-OFDM scheme, $e_{\mathrm{PAM}, n}$ also follows a Gaussian distribution.

Similar to the PAM-DMT signal, a pairwise clipping could also be employed to the ACO-OFDM signal to reduce the noise and estimation error. Due to the different symmetry structure of ACO-OFDM and PAM-DMT, we have

$$
\begin{aligned}
\hat{r}_{\mathrm{ACO}, n}^{c} & =\hat{r}_{\mathrm{ACO}, n} I_{\left\{\hat{r}_{\mathrm{ACO}, n+N / 2} \leq \hat{r}_{\mathrm{ACO}, n}\right\}}, \\
\hat{r}_{\mathrm{ACO}, n+N / 2}^{c} & =\hat{r}_{\mathrm{ACO}, n+N / 2} I_{\left\{\hat{r}_{\mathrm{ACO}, n+N / 2}>\hat{r}_{\mathrm{ACO}, n}\right\}}
\end{aligned}
$$

for $n=0, \ldots, N / 2-1$.

In the second and subsequent iterations, the pairwise clipped ACO-OFDM signal $\hat{r}_{\mathrm{ACO}, n}^{c}$ is used for detection, where we have the frequency-domain signal $\hat{R}_{\mathrm{ACO}, k}^{c}$ after FFT, and the ACO-OFDM symbols on the odd subcarriers are estimated as

$\hat{X}_{\mathrm{ACO}, k}=\arg \min _{X \in \mathcal{S}_{\mathrm{ACO}}}\left|X-2 \hat{R}_{\mathrm{ACO}, k}^{c}\right|, k=1,3, \ldots, N / 2-1$

instead of (8).

We continue the iterations and conduct another estimation of PAM-DMT symbols to further improve the performance. The iterative receiver will stop until the maximum number of iterations is reached. Compared to the conventional counterpart in [10], the complexity of iterative receiver has slightly increased, which requires one more IFFT and two pairwise clipping modules. The IFFT module has low complexity since it is usually integrated in hardware, and the pairwise clipping in (12), (13) and (17), (18) could be realized by simple comparison operations. Therefore, only slightly increased complexity will be caused.

\section{B. Power Allocation}

In HACO-OFDM, different modulation schemes are utilized in ACO-OFDM and PAM-DMT, which have different performance at the same SNR level. Specifically, PAM-DMT only employs one signal dimension of the even subcarriers, so the SNR value required for PAM-DMT to achieve the same bit error rate (BER) performance is much larger than that for ACOOFDM with the same modulation orders. Besides that, if we use different modulation orders for ACO-OFDM and PAMDMT, the required SNRs are also different. It is not desirable in practical systems since the information transmitted from both ACO-OFDM and PAM-DMT is preferred to have similar performance. Therefore, the unequal power allocation of ACO-OFDM and PAM-DMT is induced in this paper.

For ACO-OFDM with a QAM constellation of size $M_{\mathrm{ACO}}$ and PAM-DMT with a PAM constellation of size $M_{\mathrm{PAM}}$, the BER performance of QAM and PAM can be formulated as [14]

$$
P_{\mathrm{b}, \mathrm{QAM}} \approx \frac{4\left(\sqrt{M_{\mathrm{ACO}}}-1\right)}{\sqrt{M_{\mathrm{ACO}}} \log _{2}\left(M_{\mathrm{ACO}}\right)} Q\left(\sqrt{\frac{3}{M_{\mathrm{ACO}}-1} \frac{E_{s}}{N_{0}}}\right)
$$

$$
P_{\mathrm{b}, \mathrm{PAM}} \approx \frac{2\left(M_{\mathrm{PAM}}-1\right)}{M_{\mathrm{PAM}} \log _{2}\left(M_{\mathrm{PAM}}\right)} Q\left(\sqrt{\frac{6}{M_{\mathrm{PAM}}^{2}-1} \frac{E_{s}}{N_{0}}}\right)
$$

where $E_{s}$ denotes the electrical energy per symbol and $N_{0}$ represents the power spectral density of the noise. Given a required BER value $P_{b}$, we could calculate the required $E_{s} / N_{0}$ for $M_{\mathrm{ACO}}$-QAM and $M_{\mathrm{PAM}}$-PAM by numerical algorithms, then the power allocation of ACO-OFDM and PAM-DMT could be calculated by using the power allocation factor as

$$
\eta=\frac{\sqrt{E_{s, \mathrm{ACO}}}}{\sqrt{E_{s, \mathrm{ACO}}}+\sqrt{E_{s, \mathrm{PAM}}}}
$$

which denotes the proportion of optical power allocated to ACOOFDM, where $E_{s, \mathrm{ACO}}$ and $E_{s, \mathrm{PAM}}$ denote the required electrical energy per symbol of ACO-OFDM and PAM-DMT to achieve the required BER.

\section{Simulation Results}

The BER performance of the proposed iterative receiver for HACO-OFDM was evaluated by simulations, in comparison to the conventional receiver proposed in [10]. Two different HACO-OFDM modulation schemes were used, namely, ACOOFDM-4QAM with PAM-DMT-4PAM (Case 1) and ACOOFDM-16QAM with PAM-DMT-16PAM (Case 2). The size of FFT/IFFT in the transmitter was set to 512 for both ACOOFDM and PAM-DMT. We firstly generated 128 complex symbols from QAM constellations, which formed the input of the first IFFT block for ACO-OFDM. Another 127 real symbols (without the 0 -th subcarrier) were generated from 


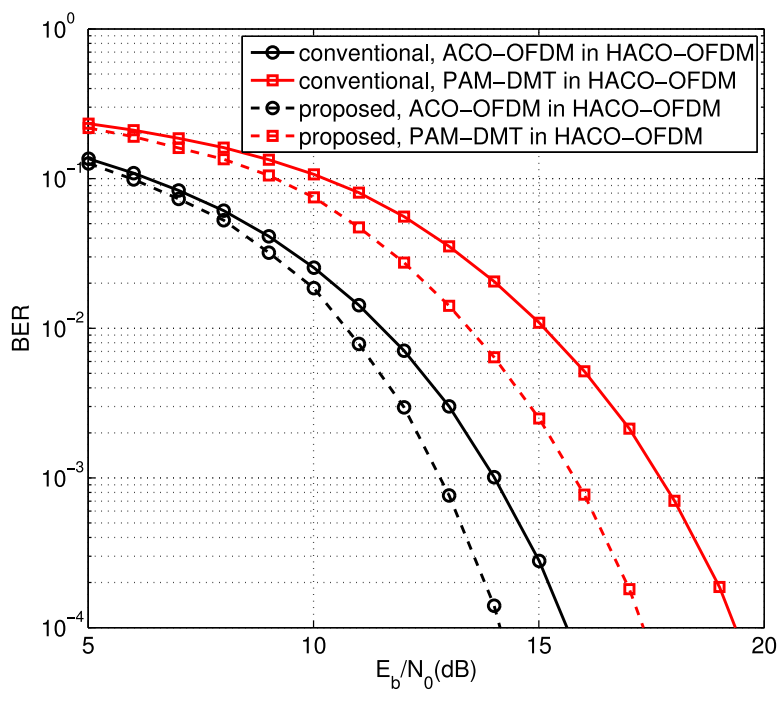

Fig. 2. BER performance comparison of the conventional receiver and our proposed iterative receiver for HACO-OFDM (Case 1 and equal power allocation).

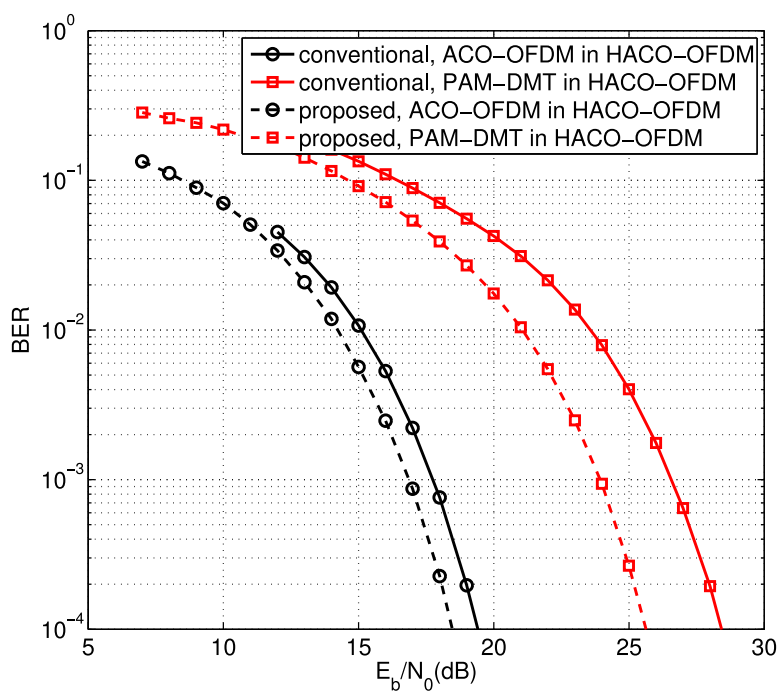

Fig. 3. BER performance comparison of the conventional receiver and our proposed iterative receiver for HACO-OFDM (Case 2 and equal power allocation).

PAM constellations which formed the input of the second IFFT block for PAM-DMT, and the output of both branches were combined as the transmitted signals in the HACO-OFDM system.

The BER performance of the proposed iterative receiver and the conventional receiver is shown in Figs. 2 and 3, where the power allocated to ACO-OFDM and PAM-DMT was assumed equal as in [10] and the iteration number of our proposed iterative receiver was set to 2 . It can be clearly seen that our proposed iterative receiver outperforms the conventional counterpart. Specifically, at BER of $10^{-3}$, our proposed receiver achieves $1.20 \mathrm{~dB}$ and $1.90 \mathrm{~dB}$ gains for ACO-OFDM and PAMDMT signals in Case 1, while the performance gains become $0.89 \mathrm{~dB}$ and $2.63 \mathrm{~dB}$ in Case 2. We could achieve more per-

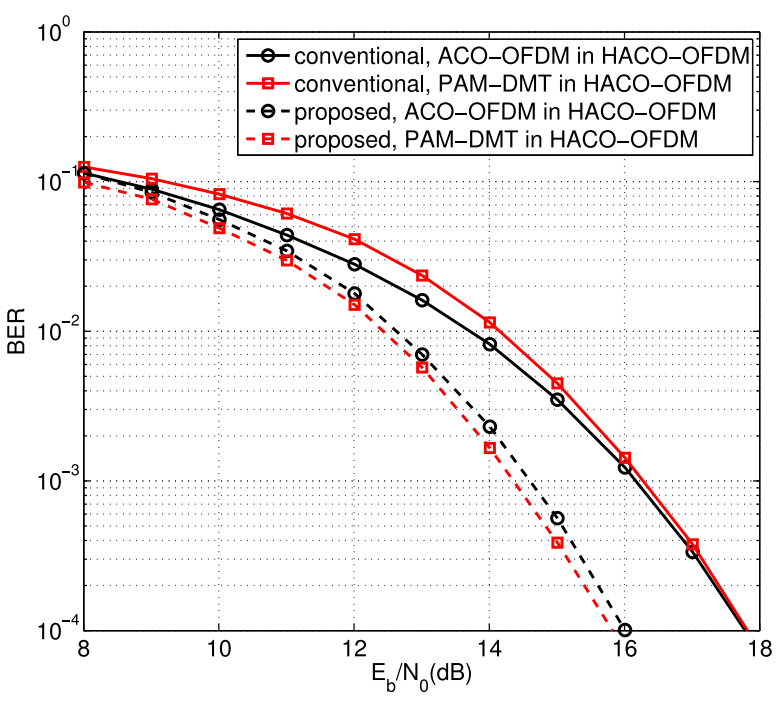

Fig. 4. BER performance comparison of the conventional receiver and our proposed iterative receiver for HACO-OFDM system (Case 1 and power allocation factor $\eta=0.3942$ ).

formance gain when $E_{b} / N_{0}$ is higher and BER is smaller since the pairwise clipping is more accurate to eliminate noise and estimation error, which is also verified in Figs. 2 and 3. At BER of $10^{-4}$, our proposed receiver achieves $1.49 \mathrm{~dB}$ and $2.04 \mathrm{~dB}$ gains for ACO-OFDM and PAM-DMT signals in Case 1 , while the performance gains become $0.94 \mathrm{~dB}$ and $2.80 \mathrm{~dB}$ in Case 2.

It also can be seen that the $E_{b} / N_{0}$ value required for achieving the BER level of $10^{-3}$ for ACO-OFDM is much smaller than that for PAM-DMT, as analyzed in Section III-B. This difference is due to the fact that PAM-DMT only employs one signal dimension of the even subcarriers and it is also interfered by the clipping noise of ACO-OFDM. At high $E_{b} / N_{0}$ regions, ACO-OFDM symbols are estimated more accurately, where the pairwise clipping operation is also more accurate. Therefore, the performance gain of our proposed iterative receiver is larger for PAM-DMT compared to that for ACO-OFDM as shown in Figs. 2 and 3.

To guarantee that ACO-OFDM and PAM-DMT signals have similar BER performance at the same $E_{b} / N_{0}$, unequal power allocation should be considered. In our simulations, the required BER was set as $P_{b}=10^{-3}$, and the corresponding power allocation factors were 0.3942 and 0.2650 in Case 1 and Case 2, respectively. The BER performance is shown in Figs. 4 and 5. It can be seen that the required $E_{b} / N_{0}$ of ACO-OFDM and PAMDMT is very close at the BER of $10^{-3}$ for both cases, which is consistent with our theoretic analysis. Our proposed iterative receiver still outperforms the conventional receiver. Specifically, at BER of $10^{-3}$, our proposed receiver achieves $1.56 \mathrm{~dB}$ and $1.91 \mathrm{~dB}$ gains for ACO-OFDM and PAM-DMT signals in Case 1 , while the performance gains become $2.05 \mathrm{~dB}$ and $2.62 \mathrm{~dB}$ in Case 2. At BER of $10^{-4}$, our proposed receiver achieves $1.78 \mathrm{~dB}$ and $2.00 \mathrm{~dB}$ gains for ACO-OFDM and PAM-DMT signals in Case 1, while the performance gains are $2.25 \mathrm{~dB}$ and $2.66 \mathrm{~dB}$ in Case 2. 


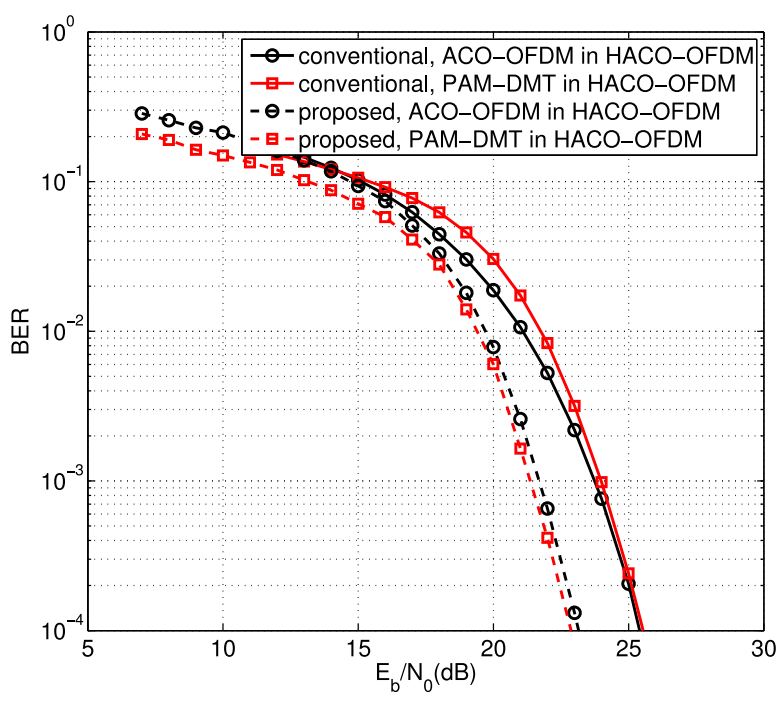

Fig. 5. BER performance comparison of the conventional receiver and our proposed iterative receiver for HACO-OFDM system (Case 2 and power allocation factor $\eta=0.2650$ ).

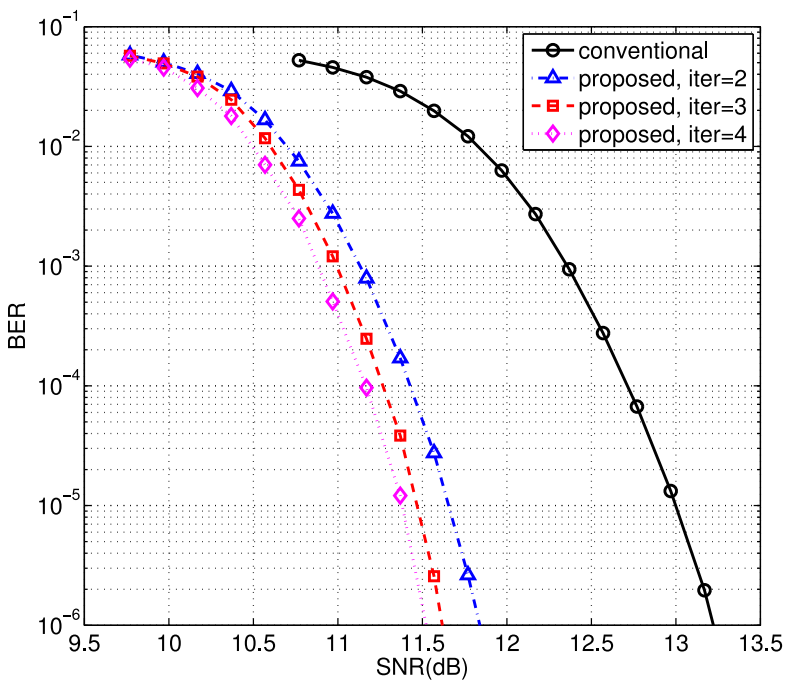

Fig. 6. BER performance comparison of the conventional receiver and our proposed iterative receiver for LDPC coded HACO-OFDM system (Case 1 and power allocation factor $\eta=0.3942$ ).

Additionally, channel coding is usually adopted in practice to further improve the system performance. For coded HACOOFDM systems, channel decoder is employed after the symbol detection, and the decoded bits are used to get more precise estimation during the iterations. In our simulations, the lowdensity parity-check (LDPC) code of the IEEE 802.11 standard with a codeword-length of 1296 bits and a code rate of 2/3 was applied [15]. The belief propagation decoder was employed for this LDPC code [16] and the maximum number of decoding iterations was set to 10 . The soft information for LDPC decoder was obtained by Max-Log-MAP algorithm, where loglikelihood ratio was calculated for each bit [17]. The power allocation factors we used were also 0.3942 and 0.2650 in Case 1 and Case 2, respectively. The detection iteration numbers of

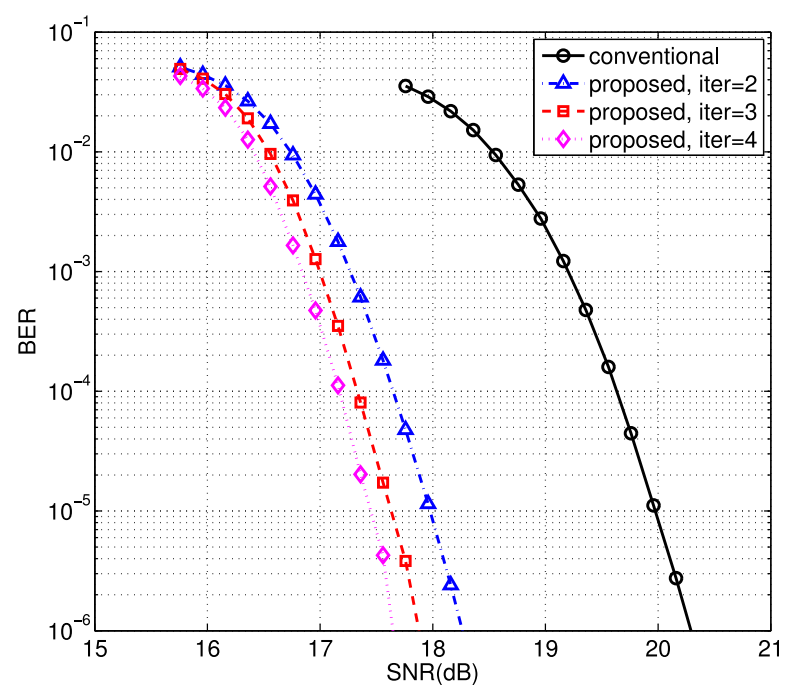

Fig. 7. BER performance comparison of the conventional receiver and our proposed iterative receiver for LDPC coded HACO-OFDM system (Case 2 and power allocation factor $\eta=0.2650$ ).

the proposed receiver were set to 2,3 , and 4 . It can be clearly seen from Figs. 6 and 7 that the coded HACO-OFDM system significantly outperforms the uncoded systems in Figs. 4 and 5, and the performance of our proposed iterative receiver is still much better than that of the conventional counterpart. At BER of $10^{-6}$, our proposed receiver achieves $1.38 \mathrm{~dB}$ after 2 detection iterations in Case 1. If we increase the detection iteration number to 3 and 4, the performance gain increases to $1.66 \mathrm{~dB}$ and $1.71 \mathrm{~dB}$. In Case 2 with higher order modulations, the performance gains are $2.03 \mathrm{~dB}, 2.41 \mathrm{~dB}$, and $2.64 \mathrm{~dB}$ when 2,3 , and 4 detection iterations are adopted. It can be seen that the performance of the proposed receiver with 4 detection iterations is very close to that with 3 detection iterations. If we further increase the detection iteration number, only small gain could be achieved at the cost of increased computational complexity and delay. Therefore, only a few detection iterations are required to achieve considerable performance gain in our proposed iterative receiver.

\section{CONCLUSION}

In this paper, an iterative receiver is proposed for HACOOFDM in IM/DD based OWC systems, where the ACO-OFDM and PAM-DMT signals are transmitted simultaneously. In our proposed receiver, ACO-OFDM and PAM-DMT signals are detected iteratively and regenerated in the time domain, so that they can be distinguished and pairwise clipping is used to reduce the effect of noise and estimation error, leading to the improved BER performance. Unequal power allocation is employed to let ACO-OFDM and PAM-DMT have similar performance at the same SNRs. Simulation results show that the proposed receiver provides significant SNR gain over the conventional receiver for both equal and unequal power allocations. For LDPC-coded HACO-OFDM systems, the proposed receiver also outperforms the conventional counterpart even with a few iterations. 


\section{REFERENCES}

[1] A. Jovicic, J. Li, and T. Richardson, "Visible light communication: Opportunities, challenges and the path to market," IEEE Commun. Mag., vol. 51, no. 12, pp. 26-32, Dec. 2013.

[2] A. M. Khalid, G. Cossu, R. Corsini, P. Choudhury, and E. Ciaramella, "1-Gb/s transmission over a phosphorescent white LED by using rateadaptive discrete multitone modulation," IEEE Photon. J., vol. 4, no. 5, pp. 1465-1473, Oct. 2012.

[3] G. Cossu, A. M. Khalid, P. Choudhury, R. Corsini, and E. Ciaramella, "3.4 Gbit/s visible optical wireless transmission based on RGB LED," Opt. Exp., vol. 20, no. 26, pp. B501-B506, Dec. 2012.

[4] S. D. Dissanayake and J. Armstrong, "Comparison of ACO-OFDM, DCOOFDM and ADO-OFDM in IM/DD systems," J. Lightw. Technol., vol. 31, no. 7, pp. 1063-1072, Apr. 2013.

[5] Z. Wang, Q. Wang, S. Chen, and L. Hanzo, "An adaptive scaling and biasing scheme for OFDM-based visible light communication systems," Opt. Exp., vol. 22, no. 10, pp. 12707-12715, May 2014.

[6] J. M. Kahn and J. R. Barry, "Wireless infrared communications," Proc. IEEE, vol. 85, no. 2, pp. 265-298, Feb. 1997.

[7] J. B. Carruthers and J. M. Kahn, "Multiple-subcarrier modulation for nondirected wireless infrared communication," IEEE J. Sel. Areas Commun., vol. 14, no. 3, pp. 538-546, Apr. 1996.

[8] J. Armstrong and A. J. Lowery, "Power efficient optical OFDM," Electron. Lett., vol. 42, no. 6, pp. 370-372, Mar. 2006.

[9] S. C. J. Lee, S. Randel, F. Breyer, and A. M. J. Koonen, "PAM-DMT for intensity-modulated and direct-detection optical communication systems," IEEE Photon. Technol. Lett., vol. 21, no. 23, pp. 1749-1751, Dec. 2009.

[10] B. Ranjha and M. Kavehrad, "Hybrid asymmetrically clipped OFDMbased IM/DD optical wireless system," J. Opt. Commun. Netw., vol. 6, no. 4, pp. 387-396, Apr. 2014.

[11] J. Armstrong, "OFDM for optical communications," J. Lightw. Technol., vol. 27, no. 3, pp. 189-204, Feb. 2009.

[12] Q. Wang, Z. Wang, S. Chen, and L. Hanzo, "Enhancing the decoding performance of optical wireless communication systems using receiver-side predistortion," Opt. Exp., vol. 21, no. 25, pp. 30295-30305, Dec. 2013.

[13] K. Asadzadeh, A. Dabbo, and S. Hranilovic, "Receiver design for asymmetrically clipped optical OFDM," in Proc. IEEE GLOBECOM Workshops, 2011, pp. 777-781.

[14] J. Li, X. Zhang, Q. Gao, Y. Luo, and D. Gu, "Exact BEP analysis for coherent M-ary PAM and QAM over AWGN and Rayleigh fading channels," in Proc. IEEE Veh. Technol. Conf., 2008, pp. 390-394.

[15] Wireless LAN Medium Access Control and Physical Layer Specifications, IEEE Standard 802.11-2012, Mar. 2012.

[16] D. J. C. MacKay, "Good error-correcting codes based on very sparse matrices," IEEE Trans. Inf. Theory, vol. 45, no. 2, pp. 399-431, Mar. 1999.

[17] Q. Wang, Q. Xie, Z. Wang, S. Chen, and L. Hanzo, "A universal lowcomplexity symbol-to-bit soft demapper," IEEE Trans. Veh. Technol., vol. 63, no. 1, pp. 119-130, Jan. 2014.
Qi Wang received the B.S. degree from Tsinghua University, Beijing, China, in 2011. He is currently working toward the Ph.D. degree at Tsinghua National Laboratory for Information Science and Technology, Department of Electronic Engineering, Tsinghua University. His research interests include channel coding, modulation, and optical wireless communications.

Zhaocheng Wang (SM'10) received the B.S., M.S., and Ph.D. degrees from Tsinghua University, Beijing, China, in 1991, 1993, and 1996, respectively. From 1996 to 1997, he was a Postdoctoral Fellow with Nanyang Technological University, Singapore. From 1997 to 1999, he was with OKI Techno Centre Pte. Ltd., Singapore, first as a Research Engineer and then as a Senior Engineer. From 1999 to 2009, he was with SONY Deutschland GmbH, first as a Senior Engineer and then as a Principal Engineer. He is currently a Professor at the Department of Electronic Engineering, Tsinghua University. He is a holder of 33 granted US/EU patents and has published more than 50 IEEE/OSA journal papers. His research interests include wireless communications, digital broadcasting, optical wireless, and millimeter wave communications. He has served as an Associate Editor of IEEE TRANSACTIONS ON WIRELESS COMMUNICATIONS, an Associate Editor of IEEE COMMUNICATIONS LETTERS, as well as the technical program committee cochairs of many international conferences. Dr. Wang is a Fellow of the Institution of Engineering and Technology.

Linglong Dai (M'11) received the B.S. degree from Zhejiang University, Zhejiang, China, in 2003, the M.S. degree (with the highest honor) from China Academy of Telecommunications Technology, Beijing, China, in 2006, and the Ph.D. degree (with the highest honor) from Tsinghua University, Beijing, in 2011, respectively. From 2011 to 2013, he was a Postdoctoral Fellow at the Department of Electronic Engineering, Tsinghua University. Since July 2013, he has been an Assistant Professor at the Department of Electronic Engineering, Tsinghua University. His research areas include wireless communications and optical communications, with the emphasis on OFDM, MIMO, synchronization, channel estimation, multiple access techniques, and wireless positioning. He has published more than 20 journals papers and more than 10 conference papers. He also hold 10 granted patents. He received IEEE ICC Best Paper Award in 2014, URSI Young Scientists Award in 2014, National Excellent Doctora Dissertation Nomination Award in 2013, IEEE ICC Best Paper Award in 2013, Outstanding Postdoctoral Fellow of Tsinghua University in 2013, Postdoctoral Science Special Foundation of China in 2012, Excellent Doctoral Dissertation of Beijing in 2012, Outstanding Ph.D. Graduate of Tsinghua University in 2011, and Academic Star of Tsinghua University in 2011. 